\title{
EDITORIAL
}

\section{Towards the elucidation of the true impact of adipocytokines on cardiovascular risk in rheumatoid arthritis}

\author{
Patrick H Dessein ${ }^{1 *}$ and Ahmed Solomon ${ }^{2}$ \\ See related research by Kang et al. http://arthritis-research.com/content/15/6/R194
}

\begin{abstract}
Adipo(cyto)kines are mostly produced by adipose tissue and orchestrate the adverse impact of excess adiposity on cardiovascular risk. Adipokines also contribute importantly to the pathophysiology of rheumatoid arthritis. Congruent with data reported in previous investigations, Kang and colleagues report in this issue of Arthritis Research \& Therapy that adipokine concentrations are further associated with metabolic risk and inflammation and that the leptin-adiponectin ratio associates with the carotid artery resistive index in rheumatoid arthritis. Guided by evidence reported thus far on cardiovascular risk, we discuss six reasons why careful elucidation of adipokine-cardiovascular risk relations is needed in rheumatoid arthritis.
\end{abstract}

In this issue of Arthritis Research \& Therapy, Kang and colleagues investigate whether adipokines could link inflammation, metabolic risk factors and cardiovascular disease in rheumatoid arthritis (RA) [1]. Evidence in support of this paradigm was reported previously [2-6]. Patients with RA experience a markedly increased cardiovascular risk that is driven by metabolic risk factors and by high-grade inflammation [7]. Kang and colleagues measured adiponectin, leptin, resistin, tumor necrosis factor alpha and interleukin-6 concentrations and assessed the common carotid artery intima-media thickness, resistive index (RI) and plaque presence by high-resolution ultrasonography [1]. Concentrations of some of the adipokines related to inflammatory markers including C-reactive protein levels and the

\footnotetext{
* Correspondence: dessein@telkomsa.net

${ }^{1}$ Cardiovascular Pathophysiology and Genomics Research Unit, School of Physiology, Faculty of Health Sciences, University of the Witwatersrand, 7 York Road, Parktown, Johannesburg 2193, South Africa

Full list of author information is available at the end of the article
}

erythrocyte sedimentation rate, and to metabolic syndrome features.

In a previous study by our group, leptin and adiponectin concentrations were not associated with carotid intimamedia thickness and plaque [3]. In addition, the leptin-adiponectin ratio and carotid RI as markers of cardiovascular risk have not been reported in RA. For these reasons, besides the abovementioned analyses, Kang and colleagues assessed (only) the relationship of the leptin-adiponectin ratio with carotid RI. In univariate analysis, the leptin-adiponectin ratio as well as age, homeostasis model assessment for insulin resistance, waist circumference and body mass index were associated with the carotid RI. Importantly, in multivariate analysis, only age and the leptin-adiponectin ratio remained significantly related to the carotid RI. The leptin-adiponectin ratio may thus provide information about the presence of subclinical cardiovascular disease beyond that on insulin resistance as assessed by the homeostasis model of insulin resistance, as well as adiposity extent as represented by body mass index and waist circumference in RA.

Adipo(cyto)kines comprise a vast range of disparate soluble bioactive proteins that are mostly secreted by adipose tissue [8]. These molecules participate in biological processes that include inflammatory responses and thereby orchestrate the adverse impact of excess adiposity on cardiovascular risk and incident type 2 diabetes [8]. Adipokines represent both adiposity extent and biological activity. RA is a prototypic inflammatory disease. In this context, $\sim 200$ recently reported investigations substantiate an important involvement of adipokines in RA activity and severity [9]. By contrast, despite the contribution of adipokines to altered cardiovascular risk in non-RA subjects and the enhanced cardiovascular risk in RA, there is a striking paucity of reported studies on the potential role of adipokines in atherogenesis in RA. 
A myriad of pertinent reasons exist why the role of adipokines in cardiovascular risk amongst patients with RA requires thorough elucidation. First, RA can modify adipokine production $[3,9]$.

Second, and presumably more important, the presence of autoimmunity can alter the effects of adipokines on cardiovascular risk [3,4]. In non-RA subjects, adiponectin production decreases with increasing adiposity and this adipokine has anti-inflammatory properties [8]. However, in RA adiponectin has marked proinflammatory properties [9]. In fact, in Kang and colleagues' study the adiponectin concentrations were paradoxically positively associated with the erythrocyte sedimentation rate [1]. Whereas in non-RA subjects adiponectin improves metabolic risk and also directly inhibits atherogenesis, we reported recently that in RA, upon using comprehensive potential confounderadjusted analysis, adiponectin concentrations associated paradoxically with high blood pressure $[3,4]$ and in white but not black Africans with enhanced endothelial activation [4]. Endothelial activation mediates the very initial stages of atherosclerosis [3-6]. Whether such paradoxical relations represent altered effects mediated by RA or a compensatory increase in adiponectin production in the presence of heightened cardiovascular risk and in an attempt to reduce this risk needs further investigation [4].

Third, conventional risk factors and disease characteristics can impact on adipokine-atherogenesis relationships in RA [5]. Resistin concentrations thus associate independently with endothelial activation in RA, but this relation is present only in those with, and not in those without, traditional risk factors, abdominal obesity, joint damage as reflected by the presence of deformed joints or prolonged disease duration [5]. This observation further supports the need for sensitivity analysis in the present context. By contrast, interleukin- 6 concentrations are more consistently associated with endothelial activation in RA [6].

Fourth, the effects of adipokines on cardiovascular risk require examination prior to targeting the respective molecules in an attempt to reduce disease activity and severity in RA [3]. Indeed, should the protective effect of adiponectin on cardiovascular risk be preserved amongst patients with RA, then its blockade would be expected to further enhance cardiovascular risk [3].

Fifth, RA influences adiposity and its distribution, which also associates with atherosclerosis in this disease $[7,10]$.

Finally, as illustrated by the disparity in adiponectinendothelial activation relations amongst Africans previously alluded to, population origin impacts on adipokine-cardiovascular risk relations in RA [4].

A caveat of Kang and colleagues' study is that potential confounders were not systematically identified. For example, gender, cardiovascular drug use, antirheumatic agent use and the glomerular filtration rate can all influence both the concentrations and effects of adipokines [3-6]. Nevertheless, this investigation reinforces previously reported evidence that strongly suggests an intriguing and important involvement of adipokines in RA atherogenesis.

\section{Abbreviations}

RA: Rheumatoid arthritis; RI: Resistive index.

\section{Competing interests}

The authors declare that they have no competing interests.

\section{Acknowledgements}

Research performed by PHD is currently supported by the South African Medical Research Council (grant number MRC2008_DES) and the National Research Foundation.

\section{Author details}

${ }^{1}$ Cardiovascular Pathophysiology and Genomics Research Unit, School of Physiology, Faculty of Health Sciences, University of the Witwatersrand, 7 York Road, Parktown, Johannesburg 2193, South Africa. ${ }^{2}$ Department of Rheumatology, Charlotte Maxeke Johannesburg Academic Hospital, Faculty of Health Sciences, University of the Witwatersrand, 7 York Road, Parktown, Johannesburg 2193, South Africa.

Published: 17 Dec 2013

\section{References}

1. Kang Y, Park H-J, Kang M-I, Lee H-S, Lee S-W, Lee S-K, Park Y-B: Adipokines, inflammation, insulin resistance, and carotid atherosclerosis in patients with rheumatoid arthritis. Arthritis Res Ther 2013, 15:R194.

2. Gonzalez-Gay MA, Garcia-Unzueta MT, Berja A, Gonzalez-Juanatey C, Miranda-Filloy JA, Vazquez-Rodriquez TR, de Matias JM, Martin J, Dessein PH, Llorca J: Anti-TNF-alpha therapy does not modulate leptin in patients with severe rheumatoid arthritis. Clin Exp Rheumatol 2009, 27:222-228.

3. Dessein $\mathrm{PH}$, Norton GR, Badenhorst M, Woodiwiss AJ, Solomon A: Rheumatoid arthritis impacts on the independent relationships between circulating adiponectin concentrations and cardiovascular metabolic risk. Mediators Inflamm 2013, 2013:461849.

4. Dessein PH, Woodiwiss AJ, Norton GR, Tsang L, Solomon A: Independent associations of total and high molecular weight adiponectin with cardiometabolic risk and surrogate markers of enhanced early atherogenesis in black and white patients with rheumatoid arthritis: a cross-sectional study. Arthritis Res Ther 2013, 15:R128.

5. Dessein PH, Norton GR, Woodiwiss AJ, Solomon A: Independent relationship between circulating resistin concentrations and endothelial activation in rheumatoid arthritis. Ann Rheum Dis 2013, 72:1586-1588.

6. Dessein PH, Solomon A, Woodiwiss AJ, Norton GR, Tsang L, Gonzalez-Gay MA: Marked independent relationship between circulating interleukin- 6 concentrations and endothelial activation in rheumatoid arthritis. Mediators Inflamm 2013, : in press.

7. Kitas GD, Gabriel SE: Cardiovascular disease in rheumatoid arthritis: state of the art and future perspectives. Ann Rheum Dis 2011, 70:8-14.

8. Berg AH, Scherer PE: Adipose tissue, inflammation, and cardiovascular disease. Circ Res 2005, 96:939-949.

9. Gomez R, Conde J, Scotece M, Gomez-Reino JJ, Lago F, Gualillo O: What's new in our understanding of the role of adipokines in rheumatic diseases? Nat Rev Rheumatol 2011, 7:528-536.

10. Solomon A, Norton GR, Woodiwiss AJ, Dessein PH: Obesity and carotid atherosclerosis in African black and Caucasian women with established rheumatoid arthritis: a cross-sectional study. Arthritis Res Ther 2012, 14: R67

\subsection{6/ar4412}

Cite this article as: Dessein and Solomon: Towards the elucidation of the true impact of adipocytokines on cardiovascular risk in rheumatoid arthritis. Arthritis Research \& Therapy 2013, 15:127 